\title{
Ultrasonographic morphological diagnosis of chronic liver disease: 2-dimensional shear wave elastography as an add-on test
}

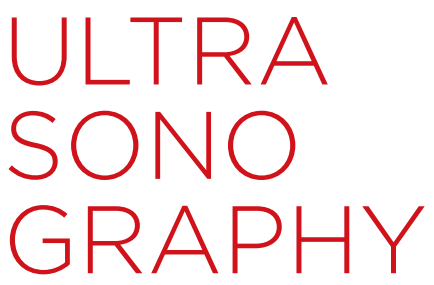

\author{
Young Seo Cho', Woo Kyoung Jeong ${ }^{2}$, Yongsoo Kim ${ }^{1}$ \\ 'Department of Radiology, Hanyang University Guri Hospital, Hanyang University College \\ of Medicine, Guri; ${ }^{2}$ Department of Radiology and Center for Imaging Sciences, Samsung \\ Medical Center, Sungkyunkwan University School of Medicine, Seoul, Korea
}

\section{ORIGINAL ARTICLE}

https://doi.org/10.14366/usg.20009 pISSN: 2288-5919 - elSSN: 2288-5943 Ultrasonography 2020;39:272-280

Purpose: This study investigated the impact of liver stiffness measurements (LSM) made using 2-dimensional (2D) shear wave elastography (SWE) on the diagnosis and grading of hepatic fibrosis and liver cirrhosis (LC) using grayscale ultrasonography (US).

Methods: This retrospective study included 46 patients who underwent liver biopsy for chronic liver disease and 33 non-biopsied subjects with no or mild fibrosis (an aspartate aminotransferase-to-platelet ratio index $<0.50$ and a Forns score $<4.21$ ). Two abdominal radiologists reviewed randomized grayscale hepatic sonogram sets with and without LSM, separated by a 4-week interval. They graded the features of echogenicity, echotexture, surface nodularity, and edge blunting and classified patients by fibrosis grade. Interobserver agreement and correlations with the fibrosis grades were compared before and after the reviewers were informed regarding LSM, and the impact of LSM on diagnostic performance was evaluated.

Results: The standard diagnoses were no or mild fibrosis ( $F 0-1, n=39)$, moderate to advanced fibrosis $(F 2-3, n=23)$, or $L C(n=17)$. The correlations between US and the diagnostic reference standard increased significantly with LSM incorporation ( 0.499 and 0.312 to 0.782 and 0.804 , $\mathrm{P}<0.01$ for both reviewers), as did interobserver agreement $(0.318$ to $0.753, \mathrm{P}<0.01)$. The areas under the receiver operating characteristic curve (AUCS) for the diagnosis of significant fibrosis increased when LSM was included ( 0.682 and 0.591 to 0.855 and $0.907, P<0.01$ for both reviewers), while the AUCs for the diagnosis of LC did not change significantly (0.891 and 0.783 to 0.904 and $0.900, P=0.849$ and $P=0.166$ ).

Conclusion: Incorporating LSM values obtained by 2D-SWE improved the diagnostic accuracy and interobserver agreement of grayscale US for hepatic fibrosis.

Keywords: Liver cirrhosis; Elastography; Hepatic fibrosis; Ultrasonography

\section{Introduction}

Ultrasonography (US) is a non-invasive modality used for imaging of the liver and biliary tract. Unlike computed tomography scanning and magnetic resonance imaging, US produces results in real time and is convenient to use at the bedside. Conventional grayscale US has been used to detect

Received: January 22, 2020

Revised: February 25, 2020

Accepted: February 29, 2020

Correspondence to:

Woo Kyoung Jeong, MD, PhD, Department of Radiology and Center for Imaging Sciences, Samsung Medical Center, Sungkyunkwan University School of Medicine, 81 Irwon-ro, Gangnam-gu, Seoul 06351, Korea

Tel. +82-2-3410-1923

Fax. +82-2-3410-0049

E-mail: jeongwk@gmail.com

This is an Open Access article distributed under the terms of the Creative Commons Attribution NonCommercial License (http://creativecommons.org/ licenses/by-nc/4.0/) which permits unrestricted noncommercial use distribution, and reproduction in any medium, provided the original work is properly cited.

Copyright (C) 2020 Korean Society of Ultrasound in Medicine (KSUM)

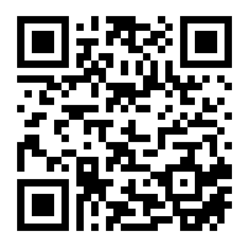

How to cite this article:

Cho YS, Jeong WK, Kim Y. Ultrasonographic morphological diagnosis of chronic liver disease: 2-dimensional shear wave elastography as an add-on test. Ultrasonography. $2020 \mathrm{Jul} ; 39(3): 272-280$.

e-ultrasonography.org 
and grade chronic liver disease (CLD) as well as to screen focal hepatic lesions. The clinical guidelines for the management of CLDs such as viral hepatitis, alcoholic liver disease, and metabolic liver disease recommend US as surveillance for liver cirrhosis (LC) and hepatocellular carcinoma [1-4]. Grayscale US findings suggestive of LC include hepatic surface nodularity, blunting of the liver edge, accentuation of the fissure, heterogeneity and coarsening of the hepatic echotexture, and morphological changes, such as enlargement of the left and caudate lobes of the liver and shrinkage of the right lobe [5]. The reported sensitivity of US in the diagnosis of $L C$ ranges from $37.5 \%$ to $95 \%$, but its sensitivity in the prediction of $C L D$ requiring clinical management (above $F 2$ and F3) is low, with values of $13.6 \%$ to $27.4 \%$, respectively. Previous studies have found no correlation between the fibrotic pattern on grayscale US and the grade of hepatic fibrosis [6-11]. In contrast, Nishiura et al. [7] used a US fibrosis scoring system involving surface nodularity, edge blunting, and echotexture assessed with low- and high-frequency probes and found that scores were significantly correlated with the histological stage of hepatic fibrosis.

Hepatic elasticity imaging is widely used for surveillance of CLD patients; recently, several research groups have published technical and clinical guidelines for CLD, including guidelines on US elastography [12-15]. Two-dimensional shear wave elastography (2D-SWE) exhibits good technical performance in the assessment of hepatic fibrosis, with a high rate of technical success and reliability [16]. In the evaluation of LC, 2D-SWE has been found to have almost the same accuracy as transient elastography (TE), the most clinically validated US elastography technique, and the correlation of 2D-SWE with the diagnosis of significant hepatic fibrosis ( $\geq F 2)$ has been found to be better than that of TE [17]. The advantage of 2D-SWE in the evaluation of hepatic fibrosis is that grayscale US examination and elasticity measurements can be performed simultaneously.

We expected that incorporating the quantitative liver stiffness measurement (LSM) obtained by 2D-SWE would increase the reliability of qualitative conventional grayscale US and the reproducibility of the diagnosis and grading of hepatic fibrosis by US. The purpose of our study was to determine the effect of the incorporation of LSM values obtained using 2D-SWE with grayscale US grading on the diagnosis of hepatic fibrosis and LC. We also compared the performance of grayscale US with and without LSM values in the diagnosis of significant fibrosis ( $\geq F 2$ ) and LC (F4) made by two independent reviewers.

\section{Materials and Methods}

\section{Patients}

This retrospective study was approved by the institutional review board of our hospital, and the requirement for informed consent was waived. The study included 61 consecutive patients who underwent percutaneous liver biopsy between September 2010 and February 2012. Most patients were examined by grayscale US and underwent LSM measurement by 2D-SWE prior to biopsy, but 15 patients did not undergo LSM measurement and were excluded. The causes of liver biopsy and LSM evaluation were alcoholic $L C(n=9)$, viral hepatitis $B(n=12)$, viral hepatitis $C(n=11)$, co-infection with hepatitis $B$ and $C(n=1)$, heavy alcohol consumption with underlying viral hepatitis $B(n=2)$, suspicion of autoimmune hepatitis $(n=4)$, non-alcoholic fatty liver disease $(n=1)$, fever of unknown origin $(n=1)$, and cryptogenic $L C(n=1)$.

In the present study, the subjects were classified into three groups: no or mild fibrosis, moderate to advanced fibrosis, and LC. For recruitment of the no or mild fibrosis group, the study included 86 non-biopsied patients who underwent liver US coupled with SWE in January or February 2012. In order to include only patients with minimal hepatic fibrosis, patients with abnormal aspartate aminotransferase-to-platelet ratio indices (APRI) or Forns scores were excluded. Of the 86 patients, 37 had normal APRIs $(<0.50)$ and Forns scores $(<4.21)$. Of these, four patients with abnormal liver function profiles were also excluded. Finally, the remaining 33 subjects who did not undergo liver biopsy were assigned to the no or mild fibrosis group. The reasons for US examination of these patients were viral hepatitis $B(n=7)$, evaluation for administration of methotrexate due to autoimmune collagen vascular disease $(n=25)$, and suspicion of CLD on a previous US examination $(n=1)$.

The pathologic degree of hepatic fibrosis was graded using the Meta-Analysis of Histological Data in Viral Hepatitis (METAVIR) fibrosis scoring system. In addition to the 33 patients mentioned above, the no or mild fibrosis group included patients with scores of $\mathrm{FO}$ and $\mathrm{F} 1$ on the METAVIR scale. The moderate to advanced fibrosis group included patients with scores of $F 2$ and $F 3$, and the LC group included patients with METAVIR scores of F4. APRI and Forns scores were calculated from recorded laboratory findings and platelet counts.

\section{US Examination and Measurement of Liver Stiffness}

Grayscale US examinations and measurements of liver stiffness were performed by one of three attending abdominal radiologists. The examiners had 17, 8, and 6 years of clinical experience in abdominal radiology, and all three perform liver US as part of their regular practice. A dedicated US machine (Aixplorer, Supersonic Imagine, 
Aix-en-Provence, France) equipped with 2D-SWE functionality was used to acquire grayscale images and LSM values.

Following a routine grayscale liver US examination, LSM was measured on the right lobe of the liver with the patient in the supine position and the machine on the "not-per-oral" setting for a minimum of 8 hours. A broadband convex transducer (1-6 MHz) was positioned on an intercostal space with a good grayscale view of the liver parenchyma, and a trapezoid color box was moved on the parenchyma, positioned deeper than $2 \mathrm{~cm}$ from Glisson's capsule and away from large vessels. The scanning parameters and protocols were as follows: SWE option, standard mode; color map opacity, 50\%; displayed elasticity range, $70 \mathrm{kPa}$; smoothing factor, 5; persistence, medium mode; displayed dynamic range, $62 \mathrm{~dB}$; frame rate, 7 frames per second; mechanical index, 1.5; and thermal index of soft tissue, 1.2 to 1.4. The examiner asked patients to exhale slightly, then hold their breath for about 5 seconds, and a cine loop that included a SWE color map was obtained. Sequential frames were recalled until the examiner believed that the elasticity in the color box had reached a plateau. In addition, a circular region of interest (ROI; Q-box, Supersonic Imagine) was positioned within the color box for measurement of the mean and standard deviation of elasticity. Generally, the diameter of the ROI was $>15 \mathrm{~mm}$, but it could be changed according to the size of the measurable parenchyma. The examiners repeated the measurement sequence 5 times.

\section{Analysis of US Images}

Each set of grayscale sonograms for each patient was composed of four representative images. The key image sets of the three patient groups-no or mild fibrosis, moderate to advanced fibrosis, and LCwere randomized by another radiologist (Y.S.C.) who did not perform the US tests and was not informed regarding the clinical data of the patients. The sonograms were evaluated retrospectively in two sessions conducted more than 6 months after obtaining them. In the first session, two radiologists (W.K.J., reviewer A; Y.K., reviewer B) with 16 and 28 years of experience in abdominal imaging, respectively independently evaluated the key image sets of grayscale US while blinded to the clinical information and LSM values. They evaluated four categories of US features associated with CLD: echogenicity, echotexture, surface nodularity, and edge blunting of the liver [7]. Finally, they predicted whether patients belonged to the no or mild fibrosis, moderate to advanced fibrosis, or LC groups based on the grayscale US findings. Four weeks later, they again reviewed the image sets once informed of the LSM values, and they repeated the evaluation of the grayscale US features and prediction of the patients' fibrosis status. The reviewers knew the reference values of the LSM in advance. On the basis of a previous study, an
LSM $>7.1 \mathrm{kPa}$ was considered abnormal, while an LSM $>10.4 \mathrm{kPa}$ was considered indicative of possible LC [18].

To evaluate the grayscale US features, the two reviewers scored each finding. A score of 1 for echogenicity of the liver corresponded to homogeneity, with no difference in echogenicity between the liver parenchyma and the kidney parenchyma. A score of 3 corresponded to clearly increased echogenicity with an indefinite margin of the intrahepatic vasculature and diaphragm or to even more highly increased echogenicity. A score of 2 was given for increased echogenicity between the scores of 1 and 3 . Regarding echotexture, a score of 1 was given for a uniform smooth hepatic echotexture on US, while a score of 2 corresponded to a suspicion of mildly coarse echotexture. A score of 3 was given for a clearly coarse texture and a score of 4 for a highly coarse texture. For surface nodularity, a score of 1 indicated a smooth liver surface, a score of 2 indicated a mildly irregular surface, and a score of 3 was given for a highly irregular hepatic surface. Regarding blunting of the liver edge, a score of 1 was given for a sharp liver edge, a score of 2 for a slightly blunt tip of the edge, and a score of 3 for a blunted edge.

\section{Statistical Analysis}

The correlations between US features and fibrosis grade and between subjective final diagnosis and fibrosis grade were assessed using the Spearman test. To evaluate the interobserver variability of each US feature and the final diagnosis, the weighted kappa (K) statistic was used. A K-value of less than 0.20 indicated poor agreement, between 0.20 and 0.39 indicated fair agreement, between 0.40 and 0.59 moderate agreement, between 0.60 and 0.79 substantial agreement, and 0.80 or higher excellent agreement. The Z-test was used to evaluate differences in correlation coefficients ( $\rho$ and $k$ values). The performance of grayscale US in the diagnosis of F2-stage or higher or of F4-stage fibrosis according to the METAVIR hepatic fibrosis classification system was evaluated using receiver operating characteristic (ROC) curve analysis, and the areas under the ROC curves (AUCs) and corresponding 95\% confidence intervals were compared using the methods detailed by DeLong et al. [19]. The utility of LSM values in the diagnosis of significant fibrosis and LC was also evaluated. Statistical analyses were performed using SPSS for Windows (version 17, SPSS Inc., Chicago, IL, USA) and MedCalc for Windows (version 12.4.0.0, MedCalc Software bvba, Mariakerke, Belgium). A P-value of $<0.05$ was considered to indicate statistical significance.

\section{Results}

\section{Patient Groups and Characteristics}

Among the 46 patients who underwent liver biopsy, the numbers 
with F0, F1, F2, F3, and F4 METAVIR fibrosis scores were 3, 3, 12, 11 , and 17, respectively. The no or mild fibrosis group consisted of 39 patients: the six $\mathrm{F} 0$ and $\mathrm{F} 1$ patients and 33 patients who did not undergo liver biopsy. The 23 patients with METAVIR fibrosis scores of $F 2$ and $F 3$ were classified as the moderate to advanced fibrosis group, and the 17 patients with METAVIR fibrosis scores of F4 were classified as the LC group, which served as the reference standard (Fig. 1).

The LSM values of the total group of patients ranged from 4.0 $\mathrm{kPa}$ to $48.6 \mathrm{kPa}$, and the mean value was $11.0 \pm 8.6 \mathrm{kPa}$. The LSM values of the no or mild fibrosis group ranged from $4.0 \mathrm{kPa}$ to 9.8 $\mathrm{kPa}$, with a mean of $5.7 \pm 1.3 \mathrm{kPa}$. In the moderate to advanced fibrosis group, LSM values ranged from $6.2 \mathrm{kPa}$ to $40.2 \mathrm{kPa}$ (mean, $15.0 \pm 9.2 \mathrm{kPa}$ ), while the LSM values of the LC group ranged from 5.3 $\mathrm{kPa}$ to $48.6 \mathrm{kPa}$ (mean, $17.6 \pm 10.2 \mathrm{kPa}$ ). Patient information and laboratory findings are summarized in Table 1.

Effect of Being Informed of the LSM Values on the Correlation between US and Fibrosis Grades

The correlation coefficients between US grade and fibrosis grade are summarized in Table 2. After incorporating LSM values, the correlation coefficient between echotexture and fibrosis grade increased significantly for both reviewer A $(0.404$ to 0.697 , $P=0.007)$ and reviewer $B(0.336$ to $0.736, P=0.001)$. The correlation coefficients for reviewer $A$ for surface nodularity $(0.382$ to 0.691 , $\mathrm{P}=0.006)$ and for edge blunting (0.499 to $0.681, \mathrm{P}=0.032)$ also increased. For reviewer $B$, the correlation coefficient for edge blunting increased significantly after incorporating LSM values (0.496 to $0.783, P=0.002$ ), but the coefficient for surface nodularity did not increase to a significant extent $(0.400$ to $0.440, P=0.765)$. In the final US diagnosis, the correlation coefficients increased significantly for both reviewers $A(0.499$ to $0.782, P=0.001)$ and $B(0.312$ to $0.804, P=0.001)$ after the $L S M$ values were incorporated.

\section{Interobserver Agreement between the Two Reviewers for US Grade and Final Diagnosis}

We evaluated the changes in interobserver agreement for the US feature grade and final diagnosis by grayscale US that occurred once LSM values were included (Table 3). The interobserver agreement between reviewers A and B for echogenicity, echotexture, and edge blunting was not significantly improved by adding the LSM values. Edge blunting improved from moderate to substantial ( 0.417 to $0.621, P=0.082$ ) when the LSM values were incorporated, although not to a statistically significant degree. Only the interobserver agreement for surface nodularity was significantly improved by the addition of information regarding LSM values, from fair to moderate agreement $(0.275$ to $0.538, P=0.049)$. Knowledge of $L S M$ values significantly improved agreement on the final diagnosis of hepatic

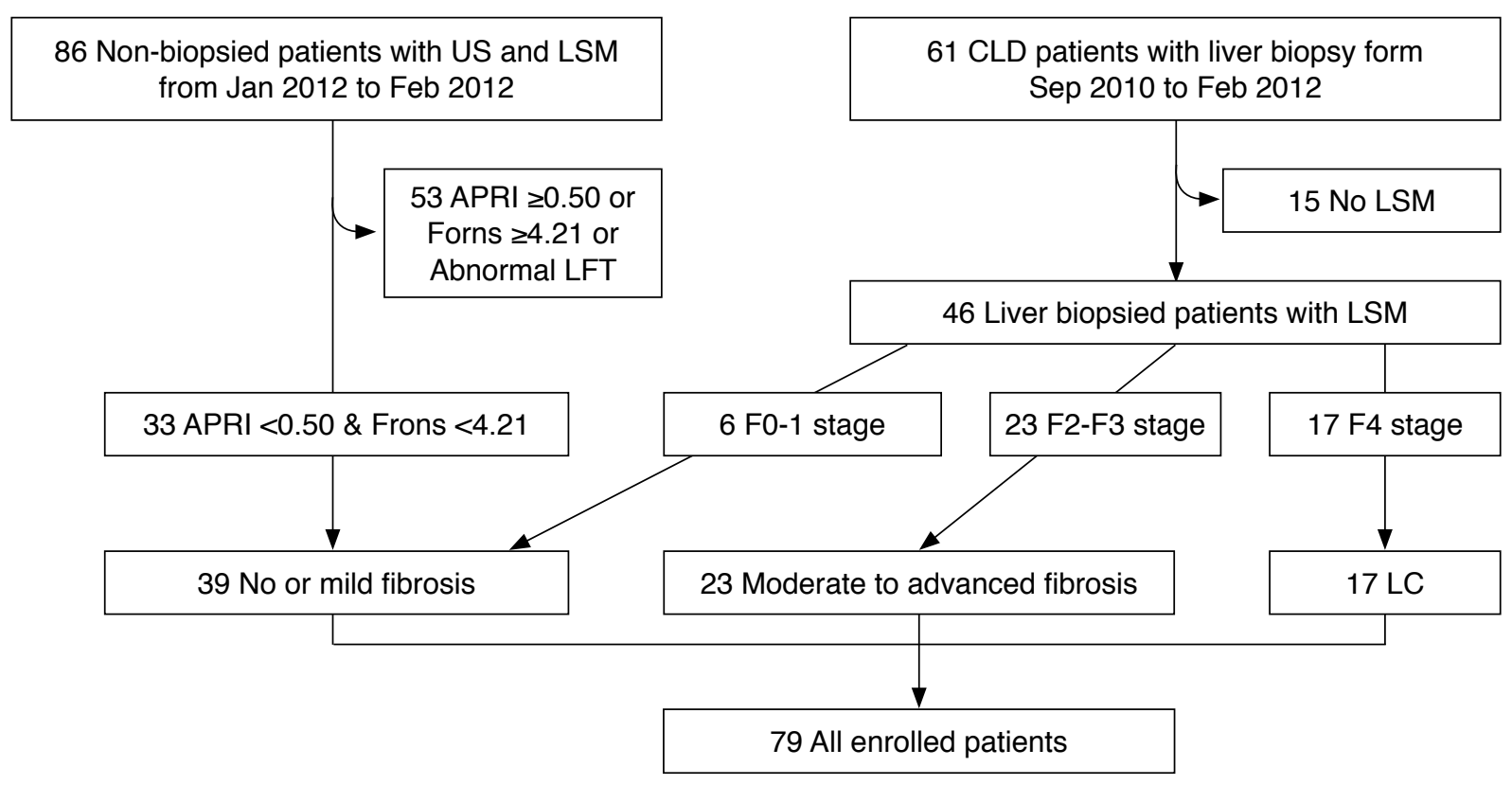

Fig. 1. Flowchart of the enrolled patients. Thirty-three patients with liver biopsy and normal aspartate aminotransferase-to-platelet ratio index (APRI) and Forns scores and 46 patients with liver biopsy were included. The enrolled patients were classified into three groups: no or mild fibrosis, moderate to advanced fibrosis, and liver cirrhosis (LC). US, ultrasonography; LSM, liver stiffness measurement; CLD, chronic liver disease; LFT, liver function test. 
Table 1. Clinical information and laboratory findings of the enrolled patients

\begin{tabular}{lcccc} 
& Total $(\mathrm{n}=79)$ & No or mild fibrosis $(\mathrm{n}=39)$ & $\begin{array}{c}\text { Moderate to advanced } \\
\text { fibrosis }(\mathrm{n}=23)\end{array}$ & $\mathrm{LC}(\mathrm{n}=17)$ \\
\hline Age $(\mathrm{yr})$ & $47.0 \pm 12.1(19-79)$ & $45.4 \pm 10.1(20-70)$ & $46.1 \pm 14.3(19-79)$ & $51.9 \pm 11.8(34-68)$ \\
Sex (M:F) & $37: 42$ & $11: 28$ & $16: 7$ & $10: 7$ \\
AST (IU/L) & $45.6 \pm 38.8(11-205)$ & $21.6 \pm 7.4(11-54)$ & $66.3 \pm 44.5(20-205)$ & $72.3 \pm 38.4(25-136)$ \\
ALT (IU/L) & $49.2 \pm 69.4(5-473)$ & $21.3 \pm 7.6(8-112)$ & $92.6 \pm 102.5(5-473)$ & $54.5 \pm 48.5(9-203)$ \\
GGT (IU/L) & $78.2 \pm 121.2(5-598)$ & $20.0 \pm 10.5(6-112)$ & $124.9 \pm 154.0(5-598)$ & $148.8 \pm 133.9(18-419)$ \\
Platelets $\left(\times 10^{3} / \mathrm{mm}^{3}\right)$ & $199.1 \pm 65.0(53-399)$ & $235.9 \pm 52.4(153-399)$ & $186.2 \pm 52.2(65-275)$ & $123.3 \pm 45.0(53-230)$ \\
APRI & $0.75 \pm 0.89(0.12-5.52)$ & $0.24 \pm 0.09(0.12-0.61)$ & $1.03 \pm 0.77(0.20-2.89)$ & $1.54 \pm 1.24(0.49-5.52)$ \\
Forns score & $5.00 \pm 2.35(1.49-12.41)$ & $3.42 \pm 0.82(1.50-5.59)$ & $5.63 \pm 2.27(1.49-10.98)$ & $7.77 \pm 1.64(5.21-12.41)$ \\
LSM (kPa) & $11.0 \pm 8.6(4.0-48.6)$ & $5.7 \pm 1.3(4.0-9.8)$ & $15.0 \pm 9.2(6.2-40.2)$ & $17.6 \pm 10.2(5.3-48.6)$ \\
\hline
\end{tabular}

Values are presented as mean \pm standard deviation (range).

LC, liver cirrhosis; AST, aspartate aminotransferase; ALT, alanine aminotransferase; GGT, gamma-glutamyl transpeptidase; APRI, AST-to-platelet ratio index; LSM, liver stiffness measurement.

Table 2. Correlations between grayscale US grading and the reference standard with and without LSM values

\begin{tabular}{|c|c|c|c|c|c|c|}
\hline & \multicolumn{3}{|c|}{ Reviewer A } & \multicolumn{3}{|c|}{ Reviewer B } \\
\hline & Grayscale & Grayscale+LSM & P-value & Grayscale & Grayscale+LSM & P-value \\
\hline Echogenicity & 0.254 & 0.327 & 0.623 & 0.290 & 0.310 & 0.892 \\
\hline Echotexture & 0.404 & 0.697 & 0.007 & 0.336 & 0.736 & 0.001 \\
\hline Surface nodularity & 0.382 & 0.691 & 0.006 & 0.400 & 0.440 & 0.765 \\
\hline Edge blunting & 0.499 & 0.681 & 0.032 & 0.496 & 0.783 & 0.002 \\
\hline Final diagnosis & 0.499 & 0.782 & 0.001 & 0.312 & 0.804 & 0.001 \\
\hline
\end{tabular}

The Spearman test was used to evaluate correlations between the ultrasound findings and the reference standard. The Z-test was used to evaluate differences in correlation coefficients.

US, ultrasonography; LSM, liver stiffness measurement.

fibrosis based on US features from fair to substantial ( 0.318 to 0.753 , $\mathrm{P}<0.001)$.

\section{Comparison of Diagnostic Performance for Presence of $\geq \mathrm{F} 2$ and F4 Fibrosis}

In the ROC curve analysis, the AUC values for grayscale US estimation of $\geq F 2$ fibrosis by reviewers $A$ and $B$ were significantly improved by including LSM values ( 0.682 and 0.591 to 0.885 and 0.907 , respectively; $P<0.001$ for both relationships). In the diagnosis of $F 4$ fibrosis, the AUC did not increase significantly for reviewer $A(0.891$ to $0.904, P=0.849)$; for reviewer $B$, it increased more notably, but the increase was still not significant ( 0.783 to $0.900, P=0.166$ ). The AUCs for the diagnoses of $\geq F 2$ and $F 4$ fibrosis using only LSM values were 0.960 and 0.829 , respectively. These were not significantly different from the AUC values obtained using grayscale US findings and LSM values. The diagnostic performances for the estimation of $\geq \mathrm{F} 2$ and $\mathrm{F} 4$ fibrosis by the two reviewers are summarized in Table 4, and the changes in the ROC curves for grayscale US diagnosis of $\geq F 2$ and F4 fibrosis with LSM values are
Table 3. Interobserver agreement (K) between two reviewers on grayscale US with and without LSM values

\begin{tabular}{lccr}
\hline & Grayscale & Grayscale+LSM & P-value \\
\hline Echogenicity & 0.643 & 0.575 & 0.504 \\
Echotexture & 0.400 & 0.464 & 0.627 \\
Surface nodularity & 0.275 & 0.538 & 0.049 \\
Edge blunting & 0.417 & 0.621 & 0.082 \\
Final diagnosis & 0.318 & 0.753 & $<0.001$ \\
\hline
\end{tabular}

The weighted $\mathrm{k}$ statistic was used to evaluate interobserver agreement. The Z-test was used to evaluate differences in correlation coefficients.

US, ultrasonography; LSM, liver stiffness measurement.

shown in Fig. 2.

\section{Discussion}

Grayscale US is the first-line imaging method for patients with chronic liver diseases. It is inexpensive and has minimal adverse biological effects $[20,21]$. However, evaluation of the liver parenchyma using grayscale US is relatively subjective, 
Table 4. Diagnostic performance of LSM alone and grayscale US with and without LSM for estimating $\geq F 2$ and F4 fibrosis by each reviewer: receiver operating characteristic analysis

\begin{tabular}{|c|c|c|c|c|c|c|c|}
\hline Parameter & AUC & $95 \% \mathrm{Cl}$ & P-value & $\begin{array}{l}\text { Sensitivity } \\
(95 \% \mathrm{Cl}, \%)\end{array}$ & $\begin{array}{l}\text { Specificity } \\
(95 \% \mathrm{Cl}, \%)\end{array}$ & $\begin{array}{c}\text { Difference, } \\
\text { P-value }^{\text {a) }}\end{array}$ & $\begin{array}{l}\text { Differences from } \\
\text { LSM, P-value }^{\text {b) }}\end{array}$ \\
\hline \multicolumn{8}{|l|}{$\geq F 2$} \\
\hline LSM & 0.960 & $0.889-0.991$ & $<0.001$ & 87.5 (73.2-95.8) & $94.9(82.6-99.2)$ & & - \\
\hline \multicolumn{8}{|l|}{ Reviewer A } \\
\hline Grayscale & 0.682 & $0.568-0.782$ & 0.002 & $32.5(18.6-49.1)$ & $97.4(86.5-99.6)$ & $<0.001$ & $<0.001$ \\
\hline Grayscale & 0.591 & $0.474-0.700$ & 0.157 & $12.5(4.2-26.8)$ & $100.0(90.9-100.0)$ & $<0.001$ & $<0.001$ \\
\hline Grayscale+LSM & 0.907 & $0.821-0.961$ & $<0.001$ & $97.5(86.8-99.6)$ & $74.4(57.9-86.9)$ & & 0.099 \\
\hline \multicolumn{8}{|l|}{ F4 } \\
\hline LSM & 0.829 & $0.728-0.905$ & $<0.001$ & $88.2(63.5-98.2)$ & $71.0(58.0-81.8)$ & - & - \\
\hline \multicolumn{8}{|l|}{ Reviewer B } \\
\hline Grayscale & 0.783 & $0.676-0.868$ & $<0.001$ & $94.1(71.2-99.0)$ & $46.8(34.0-59.9)$ & 0.166 & 0.551 \\
\hline Grayscale+LSM & 0.900 & $0.812-0.956$ & $<0.001$ & $70.6(44.1-89.6)$ & $95.2(86.5-98.9)$ & & 0.280 \\
\hline
\end{tabular}

LSM, liver stiffness measurement; US, ultrasonography; AUC, area under the receiver operating characteristic curve; $\mathrm{Cl}$, confidence interval.

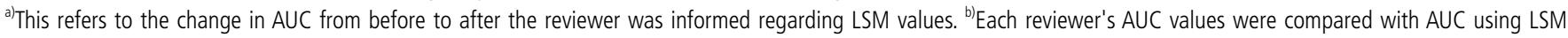
alone. The Z-test was used to evaluate differences in correlation coefficients.

and there is poor agreement between different scanners and examiners $[6,10,22]$. Of patients without secondary findings of portal hypertension who were suspected of LC based only on liver parenchymal echotexture and morphology on grayscale US, $20 \%$ actually had mild-to-moderate or no fibrosis (as evidenced by METAVIR scores of F0 to F2) on liver biopsy [23]. Even expert examiners have a tendency to overestimate $L C$, which can lead to unnecessary liver biopsy. Therefore, caution should be taken in diagnosing LC based on the liver parenchyma or morphology on grayscale US alone. Consistent US results are required to avoid confusion in the management of CLD patients in clinical practice.

In this study, the impact of including LSM values in the diagnosis of hepatic fibrosis and LC by US was evaluated. Both the correlation of grayscale US grade with the presence of hepatic fibrosis and the interobserver agreement between the two reviewers were significantly higher when LSM values were considered. Previous studies and a meta-analysis have shown LSM using 2D-SWE to have superior diagnostic accuracy to TE in the staging of hepatic fibrosis $[17,18]$. 2D-SWE has been found to have high sensitivity (approximately $90 \%$ ) in the diagnosis of clinically significant hepatic fibrosis ( $\geq F 2)$ or $L C(F 4)$, while grayscale US has been shown to exhibit sensitivity between $25 \%$ and $80 \%$ even with the use of a complex scoring system. Additionally, 2D-SWE is a reliable and reproducible method for assessing liver elasticity [24]. We obtained high AUC values of 0.960 and 0.829 when significant fibrosis $(\geq F 2)$ and LC (F4) were predicted using LSM. Therefore, the incorporation of LSM values is expected to improve not only the consistency of the US findings but also interobserver agreement in the grading of hepatic fibrosis.

Except for hepatic echogenicity, which correlates with hepatic steatosis, grayscale US findings related to hepatic fibrosisechotexture, surface nodularity, and edge blunting-yielded generally improved interobserver agreement and correlation with the reference standard when LSM values were included, although the correlations varied between reviewers. In previous studies, surface nodularity was the most sensitive grayscale US feature in the detection of significant fibrosis when a high-frequency probe was used [25]. A US scoring system involving grayscale US results obtained with a high-frequency probe exhibited higher sensitivity than TE in the diagnosis of LC [26]. Nevertheless, a meta-analysis of previous studies found sensitivity varying from $12.5 \%$ to $87.5 \%$ [27]. Liver surface nodularity exhibited an excellent agreement of 0.80 according to Colli et al. [6], but those authors performed examinations using a high-frequency probe. The present study yielded an interobserver agreement of 0.275 , and the correlation with the reference standard increased significantly only for reviewer 


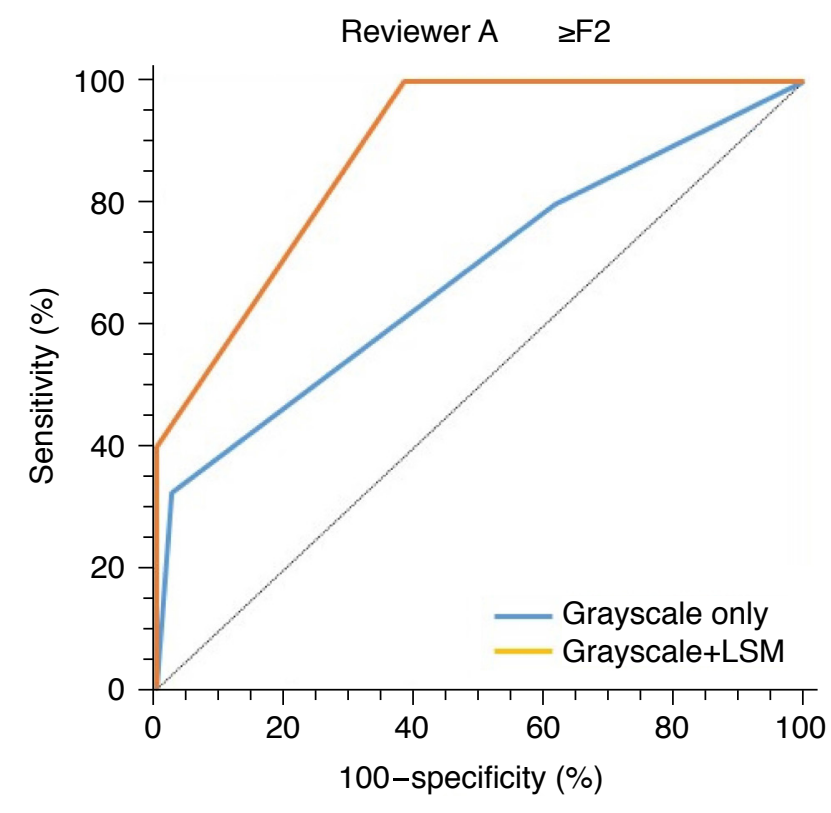

A

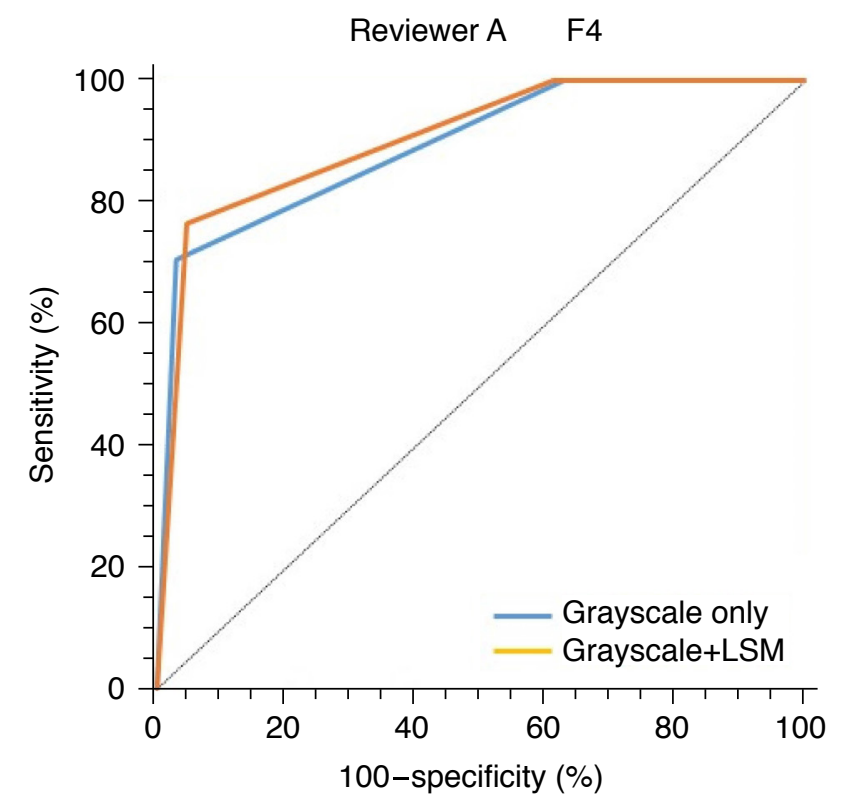

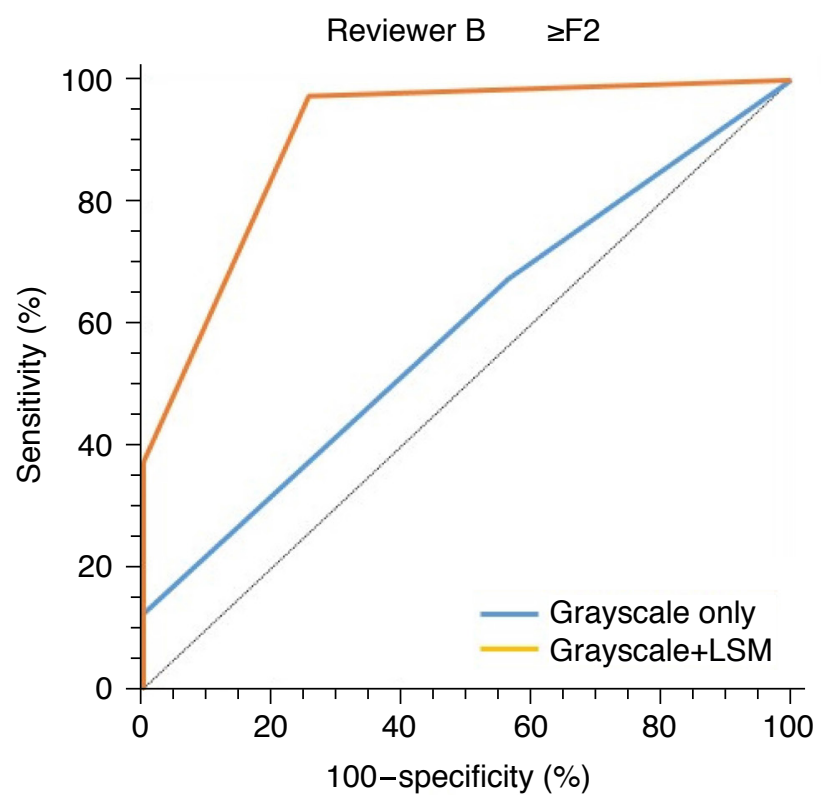

B

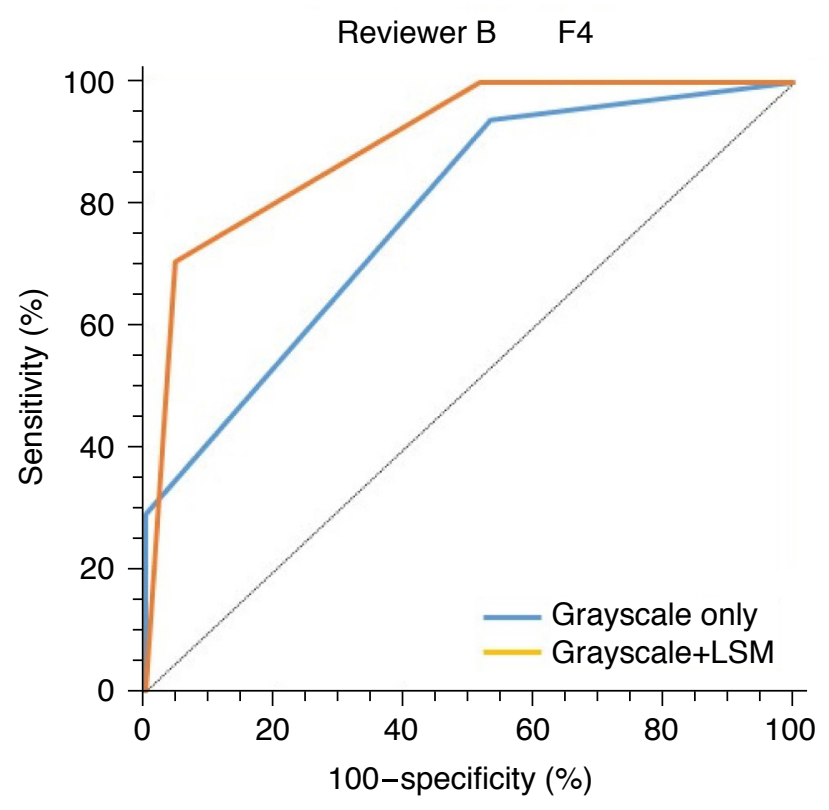

C

Fig. 2. Comparison of the receiver operating characteristic curves for diagnosis of $\geq F 2$ fibrosis and F4 fibrosis with grayscale ultrasonography (US) only and with grayscale US and knowledge of the liver stiffness measurement (LSM) values, as assigned by the two reviewers.

A, B. In the receiver operating characteristic curve analysis, the area under the receiver operating characteristic curve (AUC) values for grayscale US estimation of $\geq F 2$ fibrosis by reviewers $A$ and $B$ improved significantly when LSM values were included ( 0.682 and 0.591 to 0.885 and $0.907, \mathrm{P}<0.001$ for both relationships). C, D. In the diagnosis of F4 fibrosis, the AUC did not increase significantly for reviewer A (0.891 to $0.904 ; P=0.849$ ). For reviewer $B$, it increased more notably, but the increase was still not significant ( 0.783 to $0.900 ; P=0.166)$. LSM, liver stiffness measurement. 
A. In daily practice, low-frequency US is not sensitive as highfrequency US in the diagnosis of LC [11]. In the present study, in which a low-frequency probe was utilized, the diagnostic value of liver surface nodularity for hepatic fibrosis was considered inferior to the result expected if a high-frequency probe had been used. However, the addition of high-frequency probes to routine liver US causes examination to take longer and the process to become more complicated. In addition, such probes are difficult to utilize in patients with thick abdominal walls or large amounts of ascites, and high-frequency probes are not always available in the settings in which liver US examinations are conducted.

The combination of grayscale US and LSM in the prediction of significant fibrosis ( $\geq F 2$ ) and LC (F4) did not yield a significantly different AUC value from LSM alone. However, interobserver agreement and the diagnostic accuracy of US grading of hepatic fibrosis significantly improved with the incorporation of information about LSM values. In particular, both reviewers exhibited better discrimination between significant fibrosis $(\geq \mathrm{F} 2)$ patients and the no or mild fibrosis group, which had relatively low AUC values in the absence of LSM data. By adding 2D-SWE-based LSM measurements to routine US practice, a non-invasive reference for hepatic fibrosis could be obtained in a single session of US examination. As a result, the variability of grayscale US findings and diagnostic impressions of the degree of hepatic fibrosis could be continuously calibrated between different US examiners.

This study has several limitations. First, it was retrospective, which may have introduced selection bias, and since most of the patients in the no or mild fibrosis group did not undergo biopsy, the homogeneity of the patient group may have been impaired. Additionally, we could not evaluate grayscale US findings in real time and instead had to assess key images retrospectively, which was thus different from the clinical setting of routine US examination. Second, we did not evaluate the use of a highfrequency probe. Several studies have reported that surface nodularity using high-frequency probes was a useful finding for detecting LC on grayscale US [6]. This study involved only lowfrequency probes, so the diagnostic performance of grayscale US may have been underestimated. However, because low-frequency probes are routinely used for hepatic US examinations, our data reflect clinical US practice. Third, the CLDs of our patients had a variety of etiologies, and hepatic morphology and LSM value could not reflect the differences between the various causes of hepatic fibrosis.

In conclusion, the correlation between US findings and the reference standard, as well as interobserver agreement on the US diagnosis of hepatic fibrosis, improved when information on LSM values was incorporated. Discrimination of significant fibrosis $(\geq F 2)$ from no or mild fibrosis was also significantly improved. Thus, incorporation of LSM obtained using 2D-SWE improves the diagnostic performance of grayscale US examination of hepatic fibrosis in CLD patients.

ORCID: Young Seo Cho: https://orcid.org/0000-0003-4034-7271; Woo Kyoung Jeong: https://orcid.org/0000-0002-0676-2116; Yongs0o Kim: https://orcid.org/0000-00021069-0135

\section{Author Contributions}

Conceptualization: Jeong WK, Kim Y. Data acquisition: Jeong WK, Kim Y. Data analysis or interpretation: Cho YS, Jeong WK. Drafting of the manuscript: Cho YS. Critical revision of the manuscript: Jeong WK, Kim Y. Approval of the final version of the manuscript: all authors.

\section{Conflict of Interest}

No potential conflict of interest relevant to this article was reported.

\section{References}

1. Liaw YF. Prevention and surveillance of hepatitis B virus-related hepatocellular carcinoma. Semin Liver Dis 2005;25 Suppl 1:40-47.

2. Marrero JA, Kulik LM, Sirlin CB, Zhu AX, Finn RS, Abecassis MM, et al. Diagnosis, staging, and management of hepatocellular carcinoma: 2018 practice guidance by the American Association for the Study of Liver Diseases. Hepatology 2018;68:723-750.

3. Suk KT, Baik SK, Yoon JH, Cheong JY, Paik YH, Lee CH, et al. Revision and update on clinical practice guideline for liver cirrhosis. Korean J Hepatol 2012;18:1-21.

4. Kim YY, An C, Kim DY, Aljoqiman KS, Choi JY, Kim MJ. Failure of hepatocellular carcinoma surveillance: inadequate echogenic window and macronodular parenchyma as potential culprits. Ultrasonography 2019;38:311-320.

5. Aube C, Oberti F, Korali N, Namour MA, Loisel D, Tanguy JY, et al. Ultrasonographic diagnosis of hepatic fibrosis or cirrhosis. J Hepatol 1999;30:472-478.

6. Colli A, Fraquelli M, Andreoletti M, Marino B, Zuccoli E, Conte D. Severe liver fibrosis or cirrhosis: accuracy of US for detection: analysis of 300 cases. Radiology 2003;227:89-94.

7. Nishiura T, Watanabe H, Ito M, Matsuoka Y, Yano K, Daikoku M, et al. Ultrasound evaluation of the fibrosis stage in chronic liver disease by the simultaneous use of low and high frequency probes. Br J Radiol 2005;78:189-197.

8. Nicolau C, Bianchi L, Vilana R. Gray-scale ultrasound in hepatic cirrhosis and chronic hepatitis: diagnosis, screening, and intervention. Semin Ultrasound CT MR 2002;23:3-18.

9. Chen CH, Lin ST, Yang CC, Yeh YH, Kuo CL, Nien CK. The accuracy of sonography in predicting steatosis and fibrosis in chronic hepatitis $\mathrm{C}$. 
Dig Dis Sci 2008;53:1699-1706.

10. Kutcher R, Smith GS, Sen F, Gelman SF, Mitsudo S, Thung SN, et al. Comparison of sonograms and liver histologic findings in patients with chronic hepatitis C virus infection. J Ultrasound Med 1998;17:321-325.

11. Ong TZ, Tan HJ. Ultrasonography is not reliable in diagnosing liver cirrhosis in clinical practice. Singapore Med J 2003;44:293-295.

12. Ferraioli G, Filice C, Castera L, Choi BI, Sporea I, Wilson SR, et al. WFUMB guidelines and recommendations for clinical use of ultrasound elastography: Part 3: liver. Ultrasound Med Biol 2015;41:1161-1179.

13. de Franchis R; Baveno VI Faculty. Expanding consensus in portal hypertension: Report of the Baveno VI Consensus Workshop: Stratifying risk and individualizing care for portal hypertension. J Hepatol 2015;63:743-752.

14. European Association for Study of Liver; Asociacion Latinoamericana para el Estudio del Higado. EASL-ALEH Clinical Practice Guidelines: non-invasive tests for evaluation of liver disease severity and prognosis. J Hepatol 2015;63:237-264.

15. Dietrich CF, Bamber J, Berzigotti A, Bota S, Cantisani V, Castera L, et al. EFSUMB guidelines and recommendations on the clinical use of liver ultrasound elastography, update 2017 (long version). Ultraschall Med 2017;38:e16-e47.

16. Kim DW, Suh CH, Kim KW, Pyo J, Park C, Jung SC. Technical performance of two-dimensional shear wave elastography for measuring liver stiffness: a systematic review and meta-analysis. Korean J Radiol 2019;20:880-893.

17. Li C, Zhang C, Li J, Huo H, Song D. Diagnostic accuracy of realtime shear wave elastography for staging of liver fibrosis: a metaanalysis. Med Sci Monit 2016;22:1349-1359.

18. Ferraioli G, Tinelli C, Dal Bello B, Zicchetti M, Filice G, Filice $C$, et al. Accuracy of real-time shear wave elastography for assessing liver fibrosis in chronic hepatitis C: a pilot study. Hepatology 2012;56:2125-2133.

19. DeLong ER, DeLong DM, Clarke-Pearson DL. Comparing the areas under two or more correlated receiver operating characteristic curves: a nonparametric approach. Biometrics 1988;44:837-845.

20. Pocha C, Dieperink E, McMaken KA, Knott A, Thuras P, Ho SB. Surveillance for hepatocellular cancer with ultrasonography vs. computed tomography: a randomised study. Aliment Pharmacol Ther 2013;38:303-312.

21. Andersson KL, Salomon JA, Goldie SJ, Chung RT. Cost effectiveness of alternative surveillance strategies for hepatocellular carcinoma in patients with cirrhosis. Clin Gastroenterol Hepatol 2008;6:14181424.

22. Wu S, Tu R, Liang X. Patchy echogenicity of the liver in patients with chronic hepatitis $B$ does not indicate poorer elasticity. Ultrasonography 2019;38:327-335.

23. Kelly EM, Feldstein VA, Parks $M$, Hudock $R$, Etheridge $D$, Peters MG. An assessment of the clinical accuracy of ultrasound in diagnosing cirrhosis in the absence of portal hypertension. Gastroenterol Hepatol (N Y) 2018;14:367-373.

24. Ferraioli G, Tinelli C, Zicchetti M, Above E, Poma G, Di Gregorio M, et al. Reproducibility of real-time shear wave elastography in the evaluation of liver elasticity. Eur J Radiol 2012;81:3102-3106.

25. Choong CC, Venkatesh SK, Siew EP. Accuracy of routine clinical ultrasound for staging of liver fibrosis. J Clin Imaging Sci 2012;2:58.

26. Moon KM, Kim G, Baik SK, Choi E, Kim MY, Kim HA, et al. Ultrasonographic scoring system score versus liver stiffness measurement in prediction of cirrhosis. Clin Mol Hepatol 2013;19:389-398.

27. Allan R, Thoirs K, Phillips M. Accuracy of ultrasound to identify chronic liver disease. World J Gastroenterol 2010;16:3510-3520. 